\title{
Swarm Robotics: A Technological Advancement for Human-Swarm Interaction in Recent Era from Swarm-Intelligence Concept
}

\author{
Monalisa Hati
}

\begin{abstract}
Swarm robotics is an approach to collect robotics that takes inspiration from the self-organized behaviors of social animals. Swarm robotics is the study of how to design group of robots that operate without relying on any external infrastructure or on any form of centralized control. It is inspired from the social animals. The aim of this paper is to give a glimpse of swarm robots, algorithm for their design and applications. This paper gives a brief knowledge of the Ant colony optimization (ACO) and Particle swarm optimization (PSO) algorithm. These are the algorithms that can be used in swarm robot artificial intelligence. This paper also gives a view of the design of a robotic swarm.
\end{abstract}

Keywords: Swarm intelligence, Distributed computer architecture, Sensors

\section{Introduction}

Swarm robotics is the study of how to design group of robots that operate without relying on any external infrastructure or on any form of centralized control. Swarm robotics is an approach to collective robotics that takes inspiration from the self-organized behaviors of social animals. Through simple rules and local interactions, swarm robotics aims at designing robust, scalable and flexible collective behaviors for the coordination of large numbers of robots. swarm robotics is an advancement in an engineering field and that could be relevant to tackle real-world applications. Swarm engineering is an emerging discipline that aims at defining systematic and well founded procedures for modeling, designing, realizing, verifying, validating, operating and maintaining a swarm robotics system. . The design of robot swarms is based on Swarm intelligence principles. These principles promote the realization of systems that are fault tolerant, scalable and flexible. An object much too heavy for a single ant might not be for a group of ants working to reach a common goal. Ants are also very good at finding the fastest way to a food source from the nest by using pheromone trails. This behaviour can be applied to robotics. The key concept is to get multiple simple robots following simple rules to carry out complex tasks. This is done by building a network of communication between the individuals in a robotic swarm, to enable them to share information amongst each other. Each individual coordinates by using de-centralized control and selforganization. This would imply there is no central "brain" controlling the swarm, each robot needs to act independently . They do so by following a set of simplified rules and algorithms which produces complex swarm behaviour.

\section{Literature Review}

A swarm intelligence system usually consists of a group of simple individuals autonomously controlled by a plain set of rules and local interactions. These individuals are not necessarily unwise, but are relatively simple compared to the global intelligence achieved through the system. Some intelligent behaviors never observed in a single individual will soon emerge when several individuals begin cooperate or compete. The swarm can complete the tasks that a complex individual can do while having high robustness and flexibility and low cost. Swarm intelligence takes the full advantage of the swarm without the need of centralized control and global model, and provides a great solution for large-scale sophisticated problems. To complete a sophisticated task, a single robot must be designed with complicated structure and control modules resulting in high cost of design, construction and maintenance. Single robot is vulnerable especially when a small broken part of the robot may affect the whole system and it's difficult to predict what will happen. The swarm robotics can achieve the same ability through inter-group cooperation and takes the advantage of reusability of the simple agents and the low cost of construction and maintenance. The swarm robotics also takes the advantage of high parallelism and is especially suitable for large scale tasks. A single robot is inspired from human behaviors by comparing the corresponding nature species of these researching areas, while the swarm robotics is inspired from the social animals. Due to the restriction of current technology, it's hard to simulate the human interactions using machines or computers while the mechanisms in animal groups are easier to apply. This gives the swarm robotics a bright future in dealing with complex and large scale problems. In Swarm robotics it is supposed that a desired collective behaviour emerges from the interaction between the robots and the interaction of robots with the environment. 


\section{International Journal of Science and Research (IJSR) \\ ISSN (Online): 2319-7064}

Index Copernicus Value (2013): 6.14 | Impact Factor (2015): 6.391

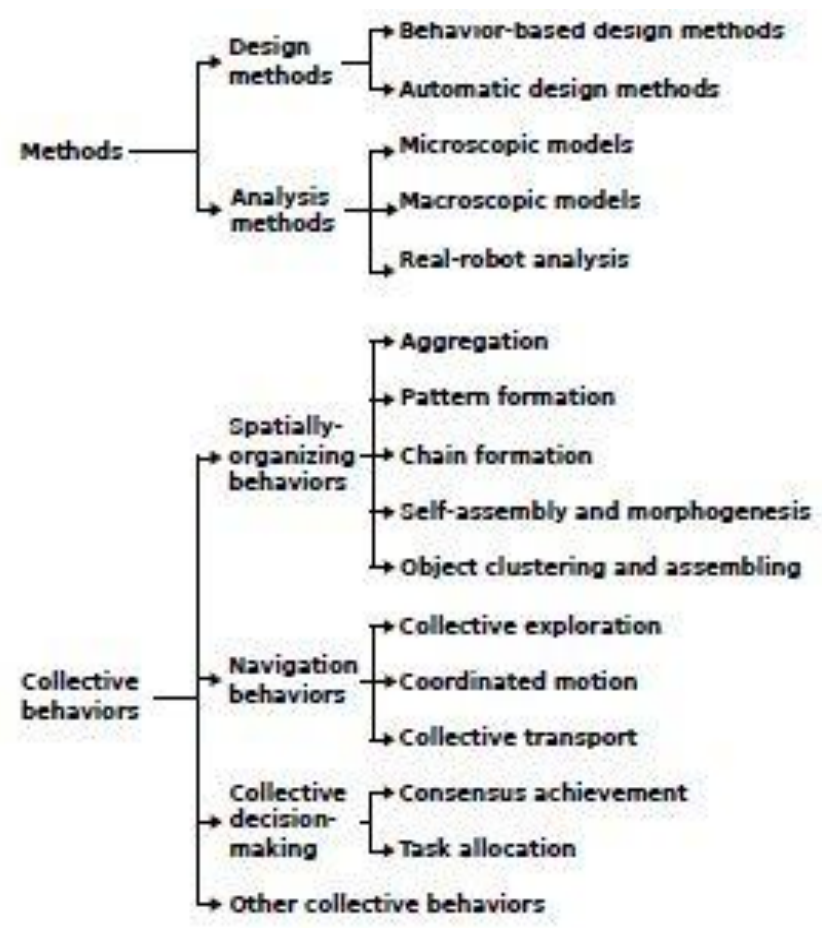

Figure1: Methods and behaviour of a Swarm-robotics Source: IRIDIA \{ Technical Report Series ISSN 1781-3794

\section{Objective}

The aim of this paper is to give a glimpse of swarm robots, algorithm for their design and applications

\section{Analysis}

The general concept of a swarm robot design is that it should be very simple and relatively cheap to produce. For example, if one robot would get lost or broken beyond repair during a mission it would not matter much. Quantity is the key to success in a robotic swarm to effectively utilize SI algorithms. A robot should also be somewhat robust, simple and effective . One example of such a robot is the swarm bot, which is also called S-bot The S-bot as the name illustrates is designed for swarm applications and is suitable for swarm functions. The S-bot is shown in [ figure 2]. The S-bot is $19 \mathrm{~cm}$ high, has a diameter of $12 \mathrm{~cm}$ and weighs around $700 \mathrm{~g}$, about the size of a handball.

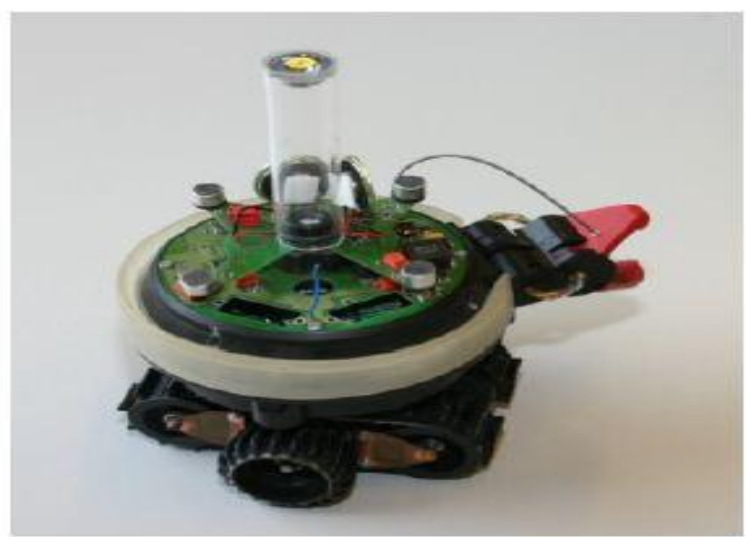

Figure 2: An Individual S-bot model

Source: Don Miner, Swarm Robotics Algorithms: A Survey

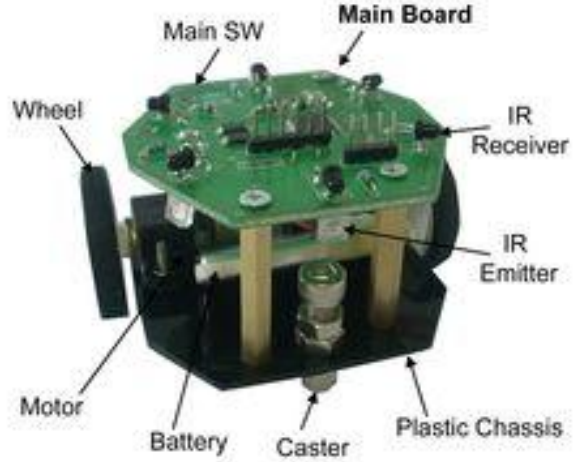

Figure 3: S-Bot and its highlighted parts

Source: www.google.com

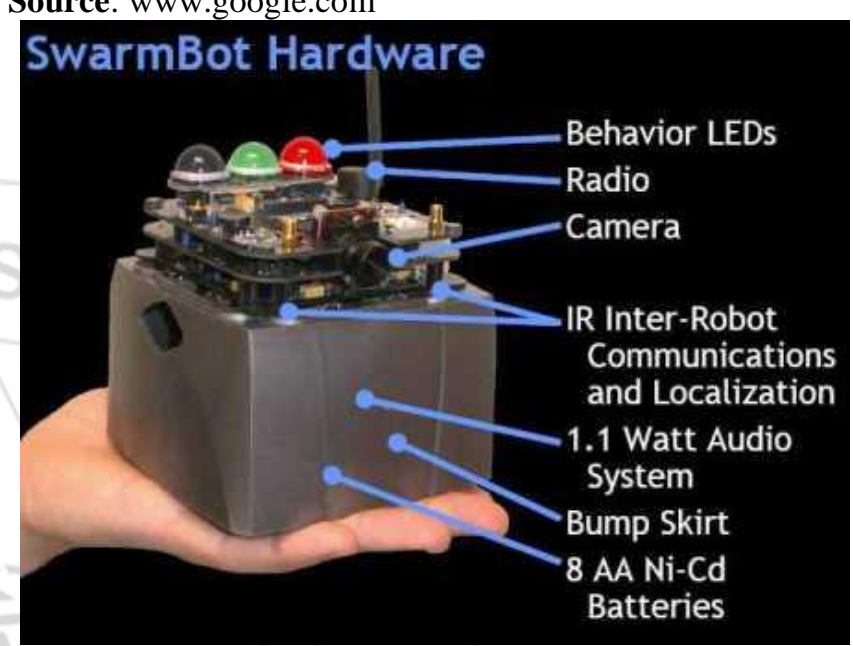

Source 4: www.google.com

The software that is needed to run robots through the exploration routine occupies $60 \mathrm{~KB}$ of memory. The S-bot utilizes a Video Graphics Array (VGA) camera for simple object recognition. To avoid collisions with other objects or sense edges the S-bot uses infrared (IR) sensors. 3 When a collision is about to occur the S-bot emits a sound to warn the other S-bots using loudspeakers. The sound emitted is then received with one of the omni-directional microphones on-board. Communication is a key factor for any robotic swarm application. This is needed to give an individual in a robotic swarm better understanding of its environment and give a better sense of orientation. To communicate with other fellow S-bots the ring mounted on the turret is of importance. The ring is equipped with eight evenly distributed multi-coloured LEDs capable of emitting red, green and blue light. Different colours emitted by the LEDring could mean different things depending on how the system is programmed. As an example red indicates a robot is seeking to maintain uniform spacing from its neighbours. Blue signals that the robot is moving into unexplored territory. Green indicates that a robot is heading back to charging dock to switch off the batteries. The bump skirt helps robots sense and avoid crashing into obstacles. S-Bots have rechargeable Ni-cd batteries and a pair of electric motors inside along with microprocessor and some associated circuitry.

A swarm it works as a collective, by a common behaviour to complete a task rather than a robot performing an 


\section{International Journal of Science and Research (IJSR) \\ ISSN (Online): 2319-7064}

Index Copernicus Value (2013): 6.14 | Impact Factor (2015): 6.391

assignment. Physical parameters are the conditions of the swarm design, though it does not play a vital role in its construction. Emphasis is put on communication due to the fact that it is the main advantage of the swarm concept. If a robot gets injured, it could call out for assistance from others in the swarm, if such approach was deemed effective. A single robot that finds a coveted object can call out for other robots in the swarm to assist in transporting of the object, if it is too heavy for one robot to move. Centralized system is used in swarm robotics communication system that deprives the swarm of flexibility and fault tolerance. In the centralized control software or a decentralized approach. The decentralized approach would have protocols to handle data fusion, swarm partitioning, energy efficiency and movement coordination. There is a need for network support such as communication load balancing, network reconfiguration, and quality of service. A functional decentralized system of this sort would result in very high fault tolerance, efficiency and flexibility, though it would still have high power consumption and need large processing capabilities. The centralized system would lower the computational and power restrictions. The flexibility and fault tolerance of a robotic swarm depends on high numbers of robots. In a system consisting of many robots working as a group the burden of performing a task does not depend on a single robot but rather depends on the group. A robotic swarm should be constructed to be able to handle a sudden loss of a robot. Therefore there should not be a single point of failure within the swarm. For a swarm robot it is essential to understand the surroundings for interaction. Navigational systems for swarms are dependent on the individual robots sensors regardless of the type of navigation. Several robots can work together to confirm a location, lowering fault probability. Robots could be designed to exchange mapping information and risk-analysis. Neural network implementations can be used to teach a robotic swarm system to behave according to given inputs from sensors and other onboard systems. Neural training is a method that could improve problem solving and efficiency in a swarm. The technique builds on the concept of one robot teaching another. A simple example of this is where positions of recharging are not known. If one robot finds a recharge station it could call out for others to gather and then lead them to the point of interest. The others could remember this point of interest, remembering the information when recharging is needed.

\footnotetext{
Algorithms:

There are two popular swarm inspired methods in swarm intelligence area. 1.Ant colony optimization (ACO) is an algorithm that can be used in swarm robot artificial intelligence. ACO algorithm is inspired by the behaviour of ants. ACO is used to solve problems regarding path finding optimizations from one place to another situated at. The general concept of ACO is not surprisingly taken from the ant world. Ants are generally very good at finding the shortest path to a food source by using pheromone trails while exploring their environment. Imagine a set of paths leading to the same food source with each path having different travelling distances to it. Ants normally start to explore their environment by choosing a random path emitting their pheromone as they go. The algorithm is as follows:
}

Input: Number of robots and no of iterations for robots to find path.

Output: Find path for the robot using highest pheromone value.

Methods:

Step1: Read the number of iteration and check how many path have to be calculated at the time of setting path.

Step2: Read the number of robots to find path accordingly the highest pheromone value

Step3: Initialization of Matrix

i. $\quad$ Set the start position value 0 .

ii. Set all the boundary values

iii. Set the value of the position of robots as X,Y,Z

iv. Set the rest of position values as zero.

Step4: Choose path for the first robot

Step5: Choose path for the second robot

Step6: Choose path for the third robot

Step7: Check the path for next robot with highest pheromone value

2. Particle swarm optimization(PSO) algorithm is inspired by the behaviour of Birds. Suppose a group of birds are randomly searching food in an area. There is only one piece of food in the area being searched. All the birds do not know where the bird is but they know how far the food is in each iteration. So the best strategy is to follow the bird which is nearest to the food. In PSO each single solution is called Particles. PSO is initialized with a group of random particles( solutions) and then searches for optima by updating generations. In every iteration, each particle is updated by following two best values. The first one is the best solution(fitness) it has achieved so far. Another best value is tracked by the particle swarm optimizer.

Pseudo code of the procedure is as follows:

For each particle

Initialize particle

END

DO

For each particle

Calculate fitness value

If the fitness value is better than the best fitness value

Set current value as the new best fitness value END

\section{Phases of Swarm robotics design:}

Algorithm Design. Swarm robotics must design both the physical robots and the behaviours of the individual robots, so the global collective behaviour emerges from their interactions.

At the moment, no general method exists to go from the individuals to the group behaviour.

Implementation and Test. The use of many real robots needs of good laboratory infrastructure to be able to perform experiments.

Analysis and Modelling. Swarm-robotic systems are usually stochastic, nonlinear, so building mathematical models for validation and optimisation is hard. These models might be necessary for creating safety real world applications 


\section{International Journal of Science and Research (IJSR) \\ ISSN (Online): 2319-7064}

Index Copernicus Value (2013): 6.14 | Impact Factor (2015): 6.391

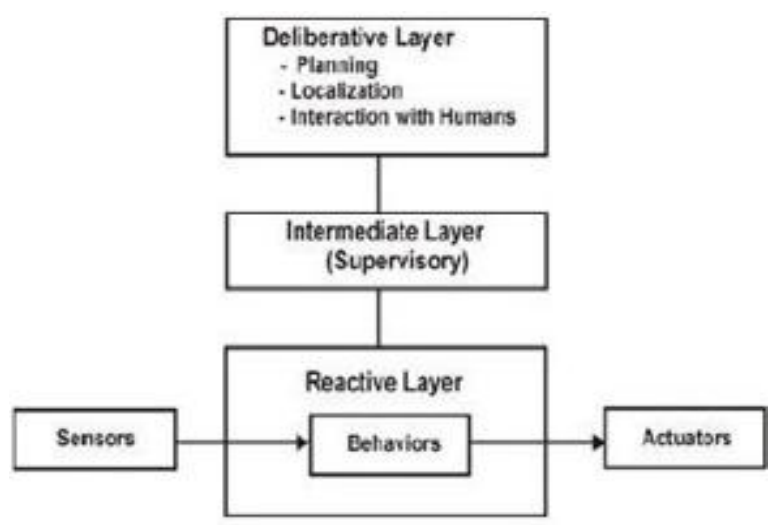

Figure 5: The three level architecture for robot control

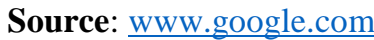

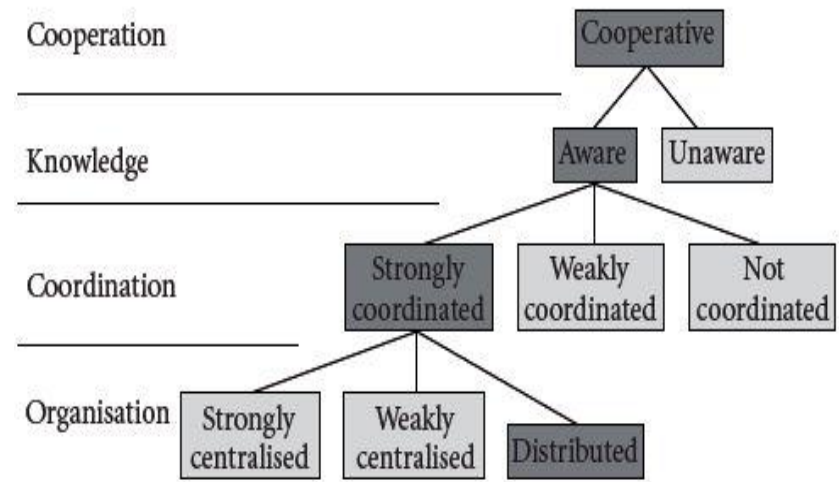

Figure 6: Taxonomy for a Swarm-robotics system.

Source: Hindawi Publishing Corporation ISRN Robotics Volume 2013, Article ID 608164, 10 pages http://dx.doi.org/10.5402/2013/608164

\section{$\underline{\text { Applications }}$}

Swarm robots can perform tasks in which the main goal is to cover a wide region. The robots can disperse and perform monitoring tasks, for example, in forests, lakes, and so forth. It can be really useful for detecting hazardous events, like a leakage of a chemical substance. The main advantage over a sensor network is that the swarm can move and focus on the problem and even act to prevent the consequences of that problem. In this way swarms of robots can be really useful for dangerous tasks. For example, for mining detection and cleaning. It can be more useful than a unique specialised robot, mainly because of the robustness of the swarm: if one robot fails and the mine explodes, the rest of the swarm continues working. In the case of a single robot this is not possible. The number of possible applications is really promising, but still the technology must be developed both in the algorithmic and modelling part, and also in the miniaturisation technologies. To reduce downtime in machinery, a robotic swarm can be used. Today much machinery needs manual inspection especially if the equipment is related to human safety. Downtime due to manual inspection can be lowered using a swarm of robots working in parallel, reaching machinery that would require cameras. Remote equipment could be reached by means of a swarm. An example of this could be power line inspection where a long distance of cable needs close and visual inspection. A swarm could travel along the power lines checking them for damage. If damage is found a larger separate robot could be sent for repairs. This would speed up the inspection process, increase efficiency and lower cost. When searching for something it often requires covering large surfaces. Whether it is under water, from the air or on the ground, more units searching imply faster cover-age due to its scalability. Here the use of robots can lower cost by replacing manned search. Were robots search with a range of sensors beyond human capabilities. This would implicate in the event that the desirable object or objects are humans that lives could be saved. These swarms could also be used in hazardous environments that are unsuitable for human interaction. Several robots also have the capability to transport objects as a group giving support one robot could not achieve. In surveillance of buildings and larger aerials, swarms give better coverage, fault tolerance and navigation than single robotic surveillance. In addition a swarm, compared to human surveillance gives lower costs.

\section{Limitations}

Swarm robotics design is more costly as the sensors, cameras, motors and other parts that make the robot work are all expensive. This also suffers from poor communication and there is lack of coordinated algorithm and applications.

\section{Conclusion}

Robotic swarm can be used in an assignment that has high cost and is difficult to perform for humans. If the assignment requires a need for scalability and fault-tolerance the gain is even higher. Swarm robot design, technology and applications are developed, enhanced and growing day by day. Swarm robots may one day be deployed by the thousands to monitor and sense the environment, inspect machinery and even perform medical procedures inside human body. Implicit communication seems to give more robustness in the communication architecture of swarm robotics. Distributed control architecture was preferred compared to centralized architecture to prevent single point failures

\section{References}

[1] M. Li, K. Lu, H. Zhu, M. Chen, S. Mao, B Prabhakaran,"Robot Swarm Communication networks: Architectures, protocols, and Appli-cations", Third International Conference on Communications and Networking in China, Hangzhou, pp.162 - 166, August 2008

[2] A Survey of Robotic Swarms by Johnny Holmström \& Daniel Romero

[3] IRIDIA Technical Report Series ISSN 1781-3794

[4] Two Foraging Algorithms for Robot Swarms Using Only Local Communication Nicholas R. Hoff III, Amelia Sagoff, Robert J. Wood, Radhika Nagpal

[5] J. Deneubourg, S. Aron, S. Goss, and J. Pasteels. The self-organising exploratory pattern of the argentine ant. Journal of Insect Behaviour, 3(2):159-168, 1990

[6] An Introduction to Swarm Robotics Inaki Navarro and Fernando Matía. Hindawi Publishing Corporation ISRN Robotics Volume 2013, Article ID 608164 